\title{
Double $\mathbf{J}$ stent prior to cystectomy increases the risk of upper urinary tract recurrence?
}

\author{
Rupesh Gupta ${ }^{1}$, Sweta Gupta ${ }^{2}$, Supriya Basu ${ }^{3}$ \\ ${ }^{1}$ Department of Urology, Mavjat Narayana Health Multispeciality Hospital, Palanpur, Gujarat, India; ${ }^{2}$ Department of Obstetrics and Gynaecology, \\ Global Hospital, Jhajjar, Haryana, India; ${ }^{3}$ Department of Urology, R. G. Kar Medical College and Hospital, Kolkata, India \\ Correspondence to: Dr. Rupesh Gupta. Consultant and Head, Department of Urology, Mavjat Narayana Health Multispeciality Hospital, Palanpur, \\ Gujarat, India. Email: drrupesgupta@gmail.com. \\ Provenance: This is a Guest Editorial commissioned by Section Editor Xiao Li (Department of Urologic Surgery, The Affiliated Cancer Hospital of \\ Jiangsu Province of Nanjing Medical University, Nanjing, China). \\ Comment on: Kiss B, Furrer MA, Wuethrich PY, et al. Stenting Prior to Cystectomy is an Independent Risk Factor for Upper Urinary Tract \\ Recurrence. J Urol 2017;198:1263-8.
}

Submitted Oct 31, 2017. Accepted for publication Nov 14, 2017.

doi: $10.21037 /$ tau. 2017.11 .26

View this article at: http://dx.doi.org/10.21037/tau.2017.11.26

Radical cystectomy is a preferred treatment of choice for the management of patients with invasive bladder cancer and for those having the superficial disease with high risk for progression. Upper urinary tract recurrence is an important complication during postoperative period after radical cystectomy. Though this is a rare complication in patients undergoing radical cystectomy for bladder cancer, it occurs in around $0.74 \%$ to $6.4 \%$ of patients and has been reported to have a poor prognosis (1).

Several risk factors have been described including age of the patient, nuclear grade, stage, multifocal or recurrent transitional cell carcinoma, history of upper urinary tract tumours, urethral or ureteral involvement, prostatic urethral involvement, lymph nodal positivity, and the presence of carcinoma in situ (CIS) for the development of upper urinary tract recurrence $(1,2)$. Therefore, there has been a growing interest in identifying predictive factors that may have a possible relationship with the increasing risk of upper urinary tract recurrence. Currently, there is no clear evidence on whether the use of double J stenting as drainage increases the risk of upper urinary tract recurrence.

Kiss et al. report their observations based on a retrospective analysis of 1,005 consecutive bladder cancer patients who underwent radical cystectomy during the 17-year period (2000 to 2016) in Bern, Switzerland (3). In this study, patients with bladder cancer were treated with radical cystectomy in the real-world scenario. The type of drainage (double $\mathrm{J}$ stent, nephrostomy or no drainage) was chosen according to the preference of the treating urologist (3).

Of the total 1,005 patients, 226 patients presented with hydronephrosis before radical cystectomy and of these 226 patients, 114 patients had preoperative drainage [double J stenting, $n=53$ (46\%); nephrostomy tube, $n=61(54 \%)$ ]. Remaining 891 patients had no preoperative drainage. Overall, seven of 53 (13\%) patients who received double J stenting developed upper urinary tract recurrence; however, none of the patients who received nephrostomy tube developed upper urinary tract recurrence. Among patients with no drainage, only 24 of 891 (3\%) developed upper urinary tract recurrence. It was also interesting to note that, all seven recurrences, among patients who had double $\mathrm{J}$ stenting, occurred within the first 2-year period and on the same side that was previously stented. One of the possible reasons specified by Kiss et al., for more number of upper urinary tract recurrences in patients with double $\mathrm{J}$ stenting include that internal ureteral stenting possibly converts a non-refluxing into a refluxing system and retrograde manipulation during stent insertion (3).

To the best of our knowledge, there is no study that demonstrated the involvement of double J sent as a risk factor for upper urinary tract recurrences in patients who underwent radical cystectomy. In a previous metaanalysis, the incidence of upper urinary tract recurrence 
after cystectomy for bladder cancer and the associated risk factors was evaluated (4). A total of 13,185 participants were included in the meta-analysis from 27 studies. Results demonstrated that multifocality increases the risk of upper urinary tract recurrence 2 to 4 times, and positive ureteral or urethral margins increase the risk sevenfold. Additionally, the risk is also increased with urothelial cancer. However, the meta-analysis did not describe the association of double J stent. Few other studies evaluated the treatment-related outcomes of ureteral stenting with an external versus double $\mathrm{J}$ stent in patients with orthotopic reservoirs after radical cystectomy $(5,6)$. In a study by Osman et al., 93 patients undergoing radical cystectomy and orthotopic neobladder were randomized in the external stent group and double $\mathrm{J}$ group (5). Two patients from each group reported the early urinary leak, only one patient from double J group developed ureteral stricture. In another study by Abdel Hakim et al., 69 patients undergoing laparoscopic radical cystectomy with orthotopic neobladder, and demonstrated that the incidence of urinary leakage was comparable in both study groups (external ureteric stent and double J stent) (6).

We would like to highlight few limitations of Kiss et al.'s study (3), only 7 patients from drainage group had developed recurrence (small numbers) though significant. Another limitation includes, this was a retrospective study which may have the inherent bias with the type of studies. Prospective studies with large sample size are warranted to confirm these results.

\section{Acknowledgements}

None.

Cite this article as: Gupta R, Gupta S, Basu S. Double J stent prior to cystectomy increases the risk of upper urinary tract recurrence? Transl Androl Urol 2017;6(6):1203-1204. doi: 10.21037/tau.2017.11.26

\section{Footnote}

Conflicts of Interest: The authors have no conflicts of interest to declare.

\section{References}

1. Kim SH, Yang HK, Lee JH, et al. A retrospective analysis of incidence and its associated risk factors of upper urinary tract recurrence following radical cystectomy for bladder cancer with transitional cell carcinoma: the significance of local pelvic recurrence and positive lymph node. PLoS One 2014;9:e96467.

2. Volkmer BG, Schnoeller T, Kuefer R, et al. Upper urinary tract recurrence after radical cystectomy for bladder cancer--who is at risk? J Urol 2009;182:2632-7.

3. Kiss B, Furrer MA, Wuethrich PY, et al. Stenting Prior to Cystectomy is an Independent Risk Factor for Upper Urinary Tract Recurrence. J Urol 2017;198:1263-8.

4. Picozzi S, Ricci C, Gaeta M, et al. Upper urinary tract recurrence following radical cystectomy for bladder cancer: a meta-analysis on 13,185 patients. J Urol 2012;188:2046-54.

5. Osman Y, Harraz AM, Barakat TS, et al. External stent versus double $\mathrm{J}$ drainage in patients with radical cystectomy and orthotopic urinary diversion: A randomized controlled trial. Int J Urol 2016;23:861-5.

6. Abdel Hakim MA, Abdalla AA, Saad IR, et al. Internal versus external ureteric stents for uretero-ileal anastomosis after laparoscopic radical cystectomy with orthotopic neobladder: A prospective comparative study. Arab J Urol 2016;14:136-42. 